\title{
Development of Maintenance Treatment Centers in Comprehensive Rehabilitation Services Clinics for Drug Abuse Based on Lifestyle Modification
}

\author{
Behzad Heydarpour ${ }^{1}$, Mozhgan Saeidi ${ }^{1}$ and Saeid Komasi ${ }^{2,{ }^{*}}$ \\ ${ }^{1}$ Cardiac Rehabilitation Center, Imam Ali Hospital, Kermanshah University of Medical Sciences, Kermanshah, Iran \\ ${ }^{2}$ Clinical Research Development Center, Imam Reza Hospital, Kermanshah University of Medical Sciences, Kermanshah, Iran \\ "Corresponding author: Clinical Research Development Center, Imam Reza Hospital, Kermanshah University of Medical Sciences, Zakarya Razi Boulevard, Kermanshah, Iran. \\ Tel/Fax: +98-8334276299, Email: s_komasi63@yahoo.com
}

Received 2018 September 24; Accepted 2018 September 28.

Keywords: Drug Abuse, Rehabilitation, Lifestyle, Services Delivery

\section{Dear Editor,}

In the recent years, maintenance treatment centers in Iran, as one of the relatively successful therapeutic protocols, have been effective in controlling substance abuse and infectious diseases (1). Nevertheless, recent reports indicate a failure of these programs and abuse recurrence in many patients $(2,3)$. Several factors, such as family, socioeconomic, environmental, cultural, and treatment-related factors can lead to treatment failure and the resumption of substance abuse in patients $(4,5)$. However, it seems that the main reason for the resumption of drug abuse by patients is the failure to adopt a healthy lifestyle (6-8).

In maintenance treatment centers, the patients refer to the center for a medical examination, drug treatment, and psychological counseling, according to a specific timetable (3). After a short time from the start of treatment, they generally refuse to participate in counseling sessions and merely refer for the medication. In other words, it seems that there is virtually no change in their lifestyle, except for the replacement of the illegal drug with the maintenance medication. In fact, psychological and environmental risk factors, malnutrition or unhealthy nutrition, physical inactivity, and weakness in stress management and family challenges are still persistent (4-8).

Based on the above, it can be said that current maintenance treatments do not cover the various aspects of an unhealthy lifestyle. These challenges make it necessary to develop maintenance treatment centers as comprehensive rehabilitation service clinics for drug abuse based on lifestyle modification. According to previous reports on the direct impact of food habits and physical activity on the recovery and treatment of substance abuse $(7,8)$, it is recommended for these components to be included in comprehensive rehabilitation services for addiction. Similar to cardiac rehabilitation programs (9), it is advisable for patients to modify unhealthy dietary habits directly under the supervision of a nutritionist. Adding a nutritionist to the rehabilitation team and directly training and supervision of the patients can facilitate their recovery. In addition, the patients can exercise at least one to three times a week (9) under the supervision of a sports medicine specialist and a physiotherapist, based on the guidelines of the American College of Sports Medicine (ACSM) (7). Exercise should include warm up, stretching, dynamic exercise, and cooling (9). Meanwhile, it is recommended that training should be provided to the patients about the physical activity duration and intensity outside the clinic. In order to enhance safety, nursing education, including blood pressure and heart rate monitoring during exercise, may be helpful. Stress management training and regular psychological counseling sessions to the patients is another important component of this comprehensive program (9).

Overall, it seems that the implementation of this practical model has an effective role in increasing the efficiency of current maintenance treatment centers. Therefore, the authors recommend setting up and implementation of comprehensive rehabilitation services for drug abuse, based on lifestyle modification, to specialists in the field. 


\section{References}

1. Keshtkaran A, Mirahmadizadeh A, Heidari A, Javanbakht M. Costeffectiveness of methadone maintenance treatment in prevention of HIV among drug users in Shiraz, south of Iran. Iran Red Crescent Med J. 2014;16(1). e7801. doi: 10.5812/ircmj.7801. [PubMed: 24719714]. [PubMed Central: PMC3964432].

2. Komasi S, Saeidi M, Soroush A. Internet-based support system and rehabilitation of drug users under maintenance treatment. Caspian J Intern Med. 2017;8(4):335-6. doi: 10.22088/cjim.8.4.335. [PubMed: 29201329]. [PubMed Central: PMC5686317].

3. Komasi S, Saeidi M, Sariaslani P, Soroush A. A new delivery model to increase adherence to methadone maintenance treatment. Caspian J Intern Med. 2018;9(1):104-5. doi: 10.22088/cjim.9.1.104. [PubMed: 29387329]. [PubMed Central: PMC5771370].

4. Komasi S, Saeidi M, Amiri MM, Nazeie N, Alizadeh NS, Soroush A. Triggers of substance abuse slip and relapse during outpatient treatment in methadone/buprenorphine maintenance therapy clinics: A predictive model with emphasis on treatment-related factors. Jundishapur J Health Sci. 2017;9(3). doi: 10.5812/jjhs.57688.
5. Ren J, Ning Z, Asche CV, Zhuang M, Kirkness CS, Ye X, et al. Trends and predictors in methadone maintenance treatment dropout in Shanghai, China: 2005-2011. Curr Med Res Opin. 2013;29(7):731-8. doi: 10.1185/03007995.2013.796284. [PubMed: 23590648].

6. Davies G, Elison S, Ward J, Laudet A. The role of lifestyle in perpetuating substance use disorder: The lifestyle balance model. Subst Abuse Treat Prev Policy. 2015;10:2. doi: 10.1186/1747-597X-10-2. [PubMed: 25595205]. [PubMed Central: PMC4326198].

7. Wang D, Wang Y, Wang Y, Li R, Zhou C. Impact of physical exercise on substance use disorders: A meta-analysis. PLoS One. 2014;9(10). e110728. doi: 10.1371/journal.pone.0110728. [PubMed: 25330437]. [PubMed Central: PMC4199732].

8. Nabipour S, Ayu Said M, Hussain Habil M. Burden and nutritional deficiencies in opiate addiction- systematic review article. Iran J Public Health. 2014;43(8):1022-32. [PubMed: 25927032]. [PubMed Central: PMC4411899].

9. Saeidi M, Soroush A, Komasi S, Singh P. A hybrid cardiac rehabilitation is as effective as a hospital-based program in reducing chest pain intensity and discomfort. Korean J Pain. 2017;30(4):265-71 doi: 10.3344/kjp.2017.30.4.265. [PubMed: 29123621]. [PubMed Central: PMC5665738]. 